\title{
Radiological Evaluation of the Initial Fixation between Cortical Bone Trajectory and Conventional Pedicle Screw Technique for Lumbar Degenerative Spondylolisthesis
}

\author{
Koshi Ninomiya, Koichi Iwatsuki, Yu-Ichiro Ohnishi, Toshiki Yoshimine \\ Department of Neurosurgery, Osaka University Graduate School of Medicine, Osaka, Japan
}

\begin{abstract}
Study Design: Retrospective study.
Purpose: To compare initial fixation using the cortical bone trajectory (CBT) technique versus conventional pedicle screws (PS) in radiographs of postsurgical lumbar degenerative spondylolisthesis.

Overview of Literature: Few reports have documented the holding strength of CBT technique for spondylolisthesis cases.

Methods: From October 2009 to June 2014, 21 cases of degenerative spondylolisthesis were surgically treated in our institution. Ten were treated with conventional PS technique and 11 of with CBT technique. Mean lumbar lordosis and percent slippage were evaluated preoperatively, immediately after surgery, and 6 months and 1 year postoperatively using radiographs. We also investigated percent loss of slip reduction.

Results: There were statistically significant differences between preoperative percent slippage and postoperative slippage in both PS and CBT procedures over 1 year, and both techniques showed good slip reduction. On the other hand, lumbar lordosis did not change significantly in either the PS or CBT groups over 1 year.

Conclusions: CBT technique showed similarly good initial fixation compared with the PS procedure in the treatment of lumbar degenerative spondylolisthesis.
\end{abstract}

Keywords: Cortical bone trajectory; Conventional pedicle screw; Lumbar degenerative spondylolisthesis

\section{Introduction}

Cortical bone trajectory (CBT) was first described by Santoni et al. [1] in 2009. Compared to traditional pedicle screw (PS) insertion, CBT is thought to be more effective for initial fixation by maximizing screw-cortical bone contact. The authors demonstrated a 30\% increase in uni- axial yield pullout load and equivalency in mixed loading for the CBT screw compared with traditional PSs in their human cadaver study.

Matsukawa et al. [2] reported a significant difference between the mean maximum intraoperative insertional torque of CBT screws and traditional screws. We also confirmed in an in vivo study that the pars interarticularis is a

Received Jul 12, 2015; Revised Aug 25, 2015; Accepted Aug 27, 2015

Corresponding author: Koshi Ninomiya

Department of Neurosurgery, Osaka University Graduate School of Medicine,

2-2 Yamadaoka, Suita 565-0871, Japan

Tel: +81-6-6879-3652, Fax: +81-6-6879-3659, E-mail: k-ninomiya@nsurg.med.osaka-u.ac.jp 
very important structure for the CBT technique in spondylolysis and non-spondylolysis cases. In the study, the mean maximum insertional torque of non-spondylolysis cases was twice as high as that of spondylolysis cases (Asian Spine Journal, in press).

However, higher pullout strength and insertional torque do not always reflect favorable initial fixation. As far as initial fixation is concerned, some postoperative radiological evaluation may be needed.

Therefore, we evaluated initial fixation through radiographs of patients with lumbar degenerative spondylolisthesis treated by the CBT technique compared to those treated by the conventional PS procedure.

\section{Materials and Methods}

Between October 2009 and June 2014, 21 patients with Meyerding grade I-II degenerative spondylolisthesis underwent posterior lumbar interbody fusion (PLIF) with conventional PS (10 patients) or CBT (11 patients) procedure in our institution. Cases of spondylolytic spondylolisthesis were excluded. All patients had lower extremity pain before surgery. We used conventional PS technique from 2009 to 2012, and CBT from 2012 to 2014. All patients had single-level fusion performed. Operative level, age, and sex distribution of the two groups did not differ (Table 1).

For evaluation of vertebral degeneration, all vertebrae on lumbar radiographs were classified using a semi-quantitative method [3]. This assessment of vertebral fractures can be performed quickly on a routine basis and cor- relates moderately well with quantitative morphometry. Vertebrae are graded on visual inspection and without direct vertebral measurement as normal (grade 0), mildly deformed (grade 1, approximately $20 \%-25 \%$ reduction in anterior, middle, and/or posterior height and a reduction of area $10 \%-20 \%$ ), moderately deformed (grade 2 , approximately $25 \%-40 \%$ reduction in any height and a reduction in area $20 \%-40 \%$ ), and severely deformed (grade 3 , approximately more than $40 \%$ reduction in any height and area). There were 7 grade 0 vertebrae and 13 grade 1 vertebrae in the PS-treated group, and 6 grade 0 vertebrae and 16 grade 1 vertebrae in the CBT-treated group; no statistical differences were evident (Table 1). There were no grade 2 and grade 3 vertebrae in this study.

\section{Surgical procedures}

The conventional PS procedure was performed in the standard fashion reported previously by Weinstein et al. [4]. Screws were 6-6.5 $\mathrm{mm}$ in diameter and $40-55 \mathrm{~mm}$ long (Easyspine multiaxial screw, Alphatec Spine, Tokyo, Japan; Capstone system screw, medtronic sofamor danek, Osaka, Japan). Two titanium (Telamon, Medtronic sofamor Danek; Novel, alphatec Spine) or polyether ether ketone (peek) (Capstone; Medtronic sofamor Danek) interbody cages were used.

CBT was performed under lateral fluoroscopy. We used the isthmus of the lamina as an anatomical landmark for entry [5]. Screws were placed $3 \mathrm{~mm}$ inside the isthmus and inserted cephalad to and laterally from the isthmus. Samesize tapping was performed. The screws were $4.5-5 \mathrm{~mm}$

Table 1. Demographic data between PS and CBT

\begin{tabular}{|c|c|c|c|}
\hline Parameter & Pedicle screws & Cortical bone trajectory & $p$-value \\
\hline No. of cases & 10 & 11 & - \\
\hline Male (\%) & 50 & 63.6 & 0.53 \\
\hline Age (yr) & $61.4 \pm 2.6$ & $62.2 \pm 2.5$ & 0.83 \\
\hline \multicolumn{4}{|l|}{ Level } \\
\hline L3-4 & 1 & 1 & 0.94 \\
\hline$\llcorner 4-5$ & 9 & 10 & \\
\hline \multicolumn{4}{|c|}{ Vertebral degeneration } \\
\hline Grade 0 & 7 & 6 & 0.59 \\
\hline Grade 1 & 13 & 16 & \\
\hline
\end{tabular}

Values of age are presented as mean \pm standard error.

PS, pedicle screws; CBT, cortical bone trajectory. 
in diameter and 2-35 $\mathrm{mm}$ in length, with a $4 \mathrm{~mm}$ pitch (Zodiac polyaxial screw, Alphatec Spine). One titanium or PEEK interbody cage (NOVEL, Alphatec Spine) was used.

In both techniques, cages were packed with autologous bone graft, and bones were also used for posterolateral bone fusion.

\section{Radiological assessment}

Radiological outcome was evaluated by comparing percent slippage and lumbar lordosis before surgery with that immediately after surgery, 6 months later, and 1 year later. Percent loss of slip reduction was also checked. Lumbar lordosis was measured from the superior end plate of L1 to the superior end plate of L5.

\section{Statistical analyses}

JMP Pro 11 (SAS Institute Inc., Cary, NC, USA) was used for statistical analyses. Statistical significance was defined as $p<0.05$. Percent slippage and lumbar lordosis were analyzed using one-way analysis of variance (ANOVA) with Tukey's test. Also, percent loss of slip was analyzed using ANOVA and the chi-square test was used for demographic data between PS and CBT.

\section{Results}

Figs. 1 and 2 depict representative cases of conventional PS and CBT techniques.

One patient treated with CBT had spacer backout 2 weeks after surgery, and he underwent reoperation. $\mathrm{He}$ was excluded from the analysis at 6 months and 1 year after the operation. Other patients had no complications and experienced a good clinical course. In conventional PS cases, lower extremity pain disappeared in $70 \%$ patients after the operation through 1 year. In CBT cases, $73 \%$ of the patients were free from pain after the operation.

Fig. 3 shows the time course of the percent slippage in each case. The percent slippage decreased from $12.7 \%$
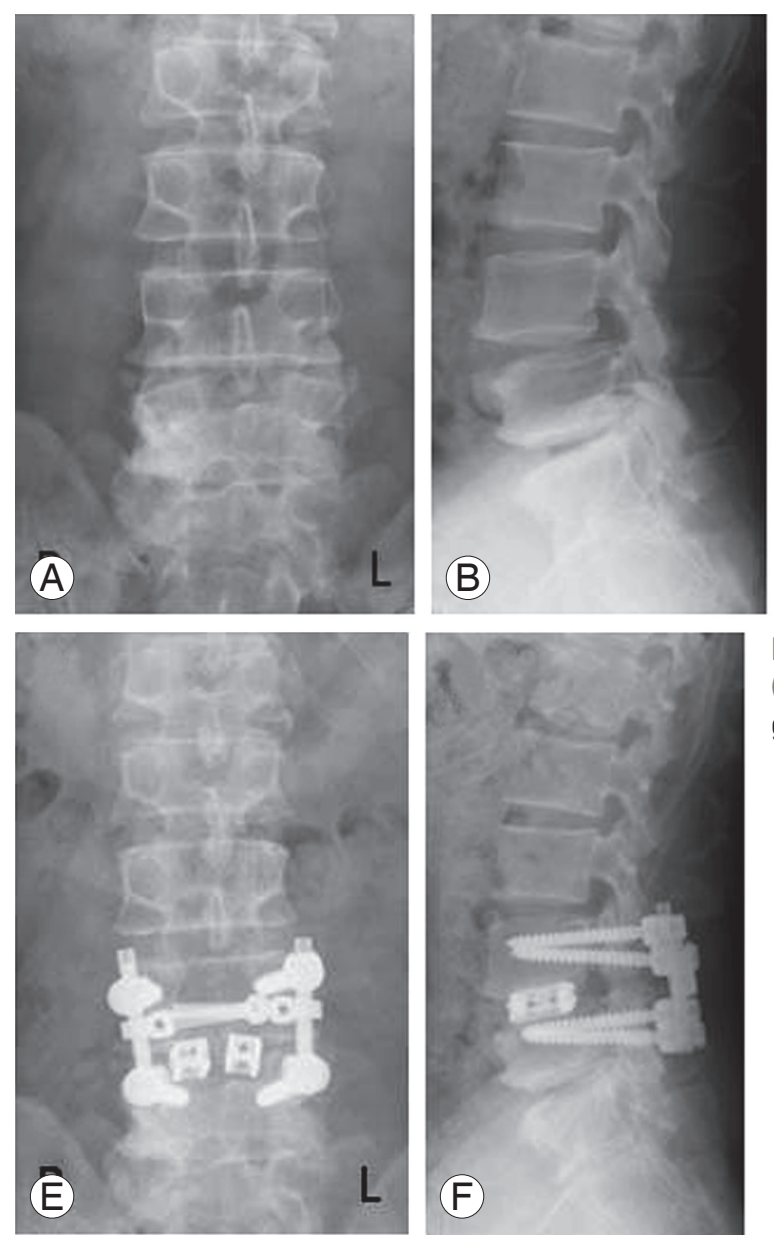
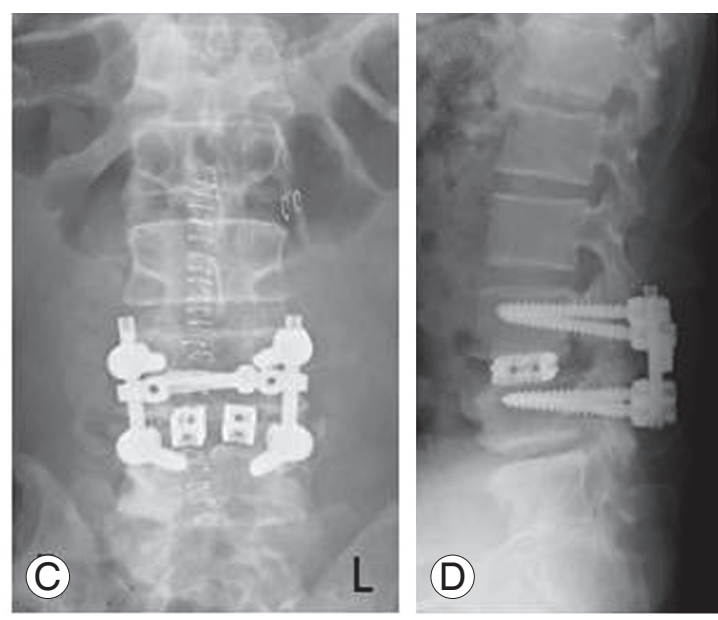

Fig. 1. Plain radiographs of a 67-year-old patient taken before $(\mathbf{A}, \mathbf{B})$, immediately after surgery (C, D), and 1 year after surgery $(E, F)$ using the conventional pedicle screws technique. 

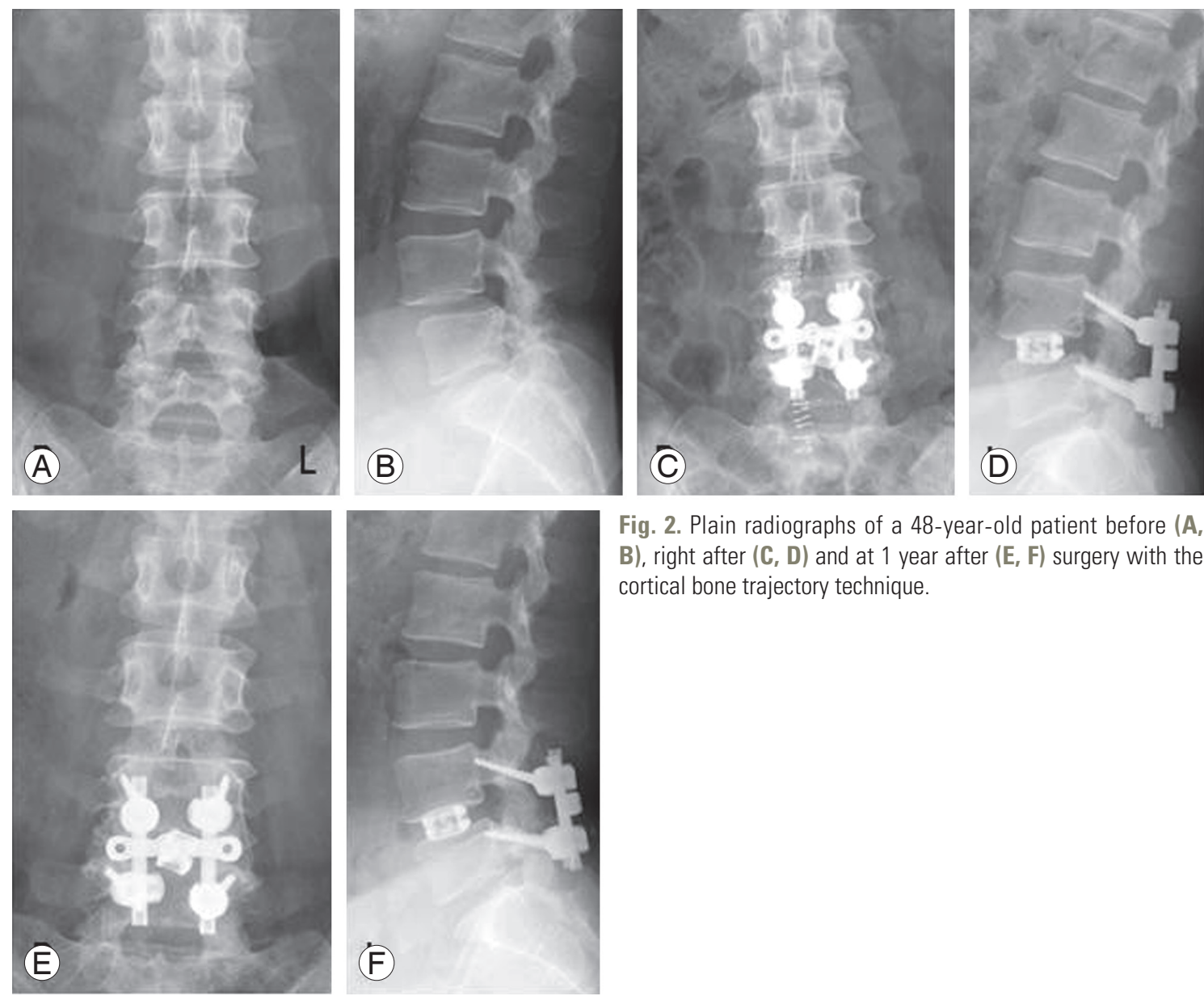

Fig. 2. Plain radiographs of a 48-year-old patient before $(\mathbf{A}$, B), right after $(\mathbf{C}, \mathbf{D})$ and at 1 year after $(\mathbf{E}, \mathbf{F})$ surgery with the cortical bone trajectory technique.
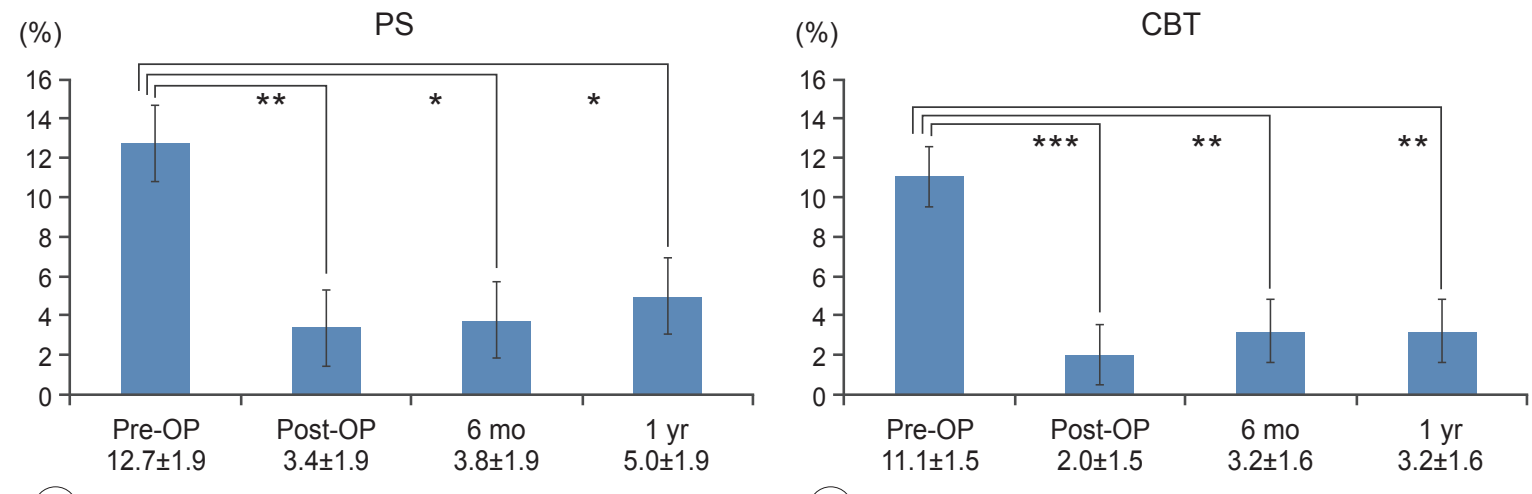
(A)

${ }^{*} p<0.05,{ }^{* *} p<0.01$

(B)

${ }^{* *} p<0.01,{ }^{* * *} p<0.001$

Fig. 3. Bar diagram showing the significant improvement of the percent slip (mean \pm standard error) with PS technique (A) and CBT technique (B). PS, pedicle screws; CBT, cortical bone trajectory; Pre-OP, preoperative; Post-OP, postoperative.

before surgery to $5.0 \%$ after 1 year using the PS technique. Using the CBT technique, the percent slippage decreased from $11.1 \%$ before surgery to $3.2 \%$ after 1 year. Both techniques demonstrated a significant difference between pre- and postoperation. In addition, there was no significant difference of loss of percent slip in 6 months and in 1 year between both procedures (Table 2, Suppl. Table 1).

The lumbar lordosis did not change significantly in ei- 
Table 2. Loss of \% slip

\begin{tabular}{lrrr} 
Time since surgery & PS $(\%)$ & CBT (\%) & $p$-value \\
$6 \mathrm{mo}$ & $0.59 \pm 0.57$ & $0.98 \pm 0.57$ & 0.63 \\
$6 \mathrm{mo}-1 \mathrm{yr}$ & $0.1 \pm 0.64$ & $0.0 \pm 0.64$ & 0.96 \\
\hline
\end{tabular}

Values are presented as mean \pm standard error.

PS, pedicle screws; CBT, cortical bone trajectory.
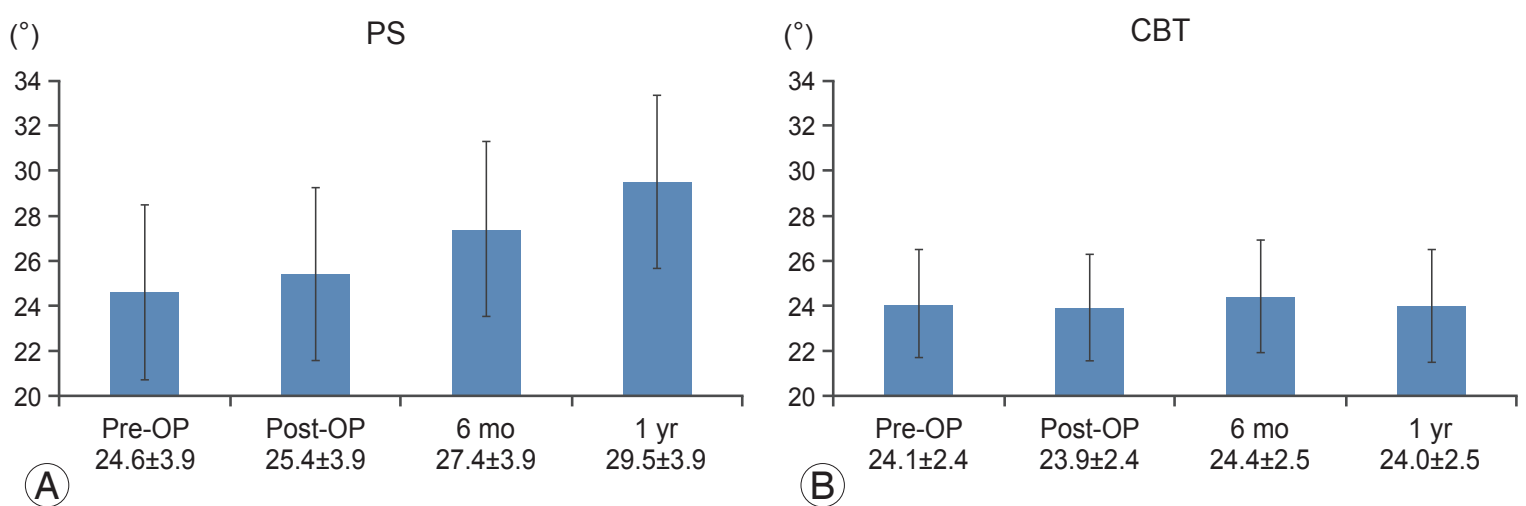

Fig. 4. Bar diagram showing that the lumbar lordosis (mean \pm standard error) did not significantly change with the PS technique (A) or CBT technique (B). PS, pedicle screws; CBT, cortical bone trajectory.

ther PS or CBT groups over 1 year (Suppl. Table 2, Fig. 4).

\section{Discussion}

CBT has a more medial-to-lateral and shorter path than the traditional technique for spinal fusion, and is thought to be effective for severely degenerated vertebrae because the screws are primarily stabilized in the posterior elements.

In vivo insertional torque of CBT screws was first reported by Matsukawa et al. [2]. The authors described a significant difference between the mean maximum insertional torque of CBT screws and traditional screws, with the former being almost twice as high as the latter.

Several studies [6-8] have reported that the insertional torque of PSs is highly correlated with pullout strength. Other studies described screw loosening caused primarily by cyclic caudocephalad toggling at the bone-screw interface [9] and that insertional torque can predict screw loosening [10].

Therefore, when we consider holding power, or initial fixation generated by PSs, we must evaluate postoperative radiographs. In the present study of degenerative spondylolisthesis cases, percent slip, loss of slip reduction, and lumbar lordotic angle were evaluated for this initial fixation.

Biomechanically, slip reduction is thought to be achieved by anteroposterior directional force, and maintenance of lordosis achieved by craniocaudal directional force. This realignment might enhance good bone fusion and clinical outcome [11], and prevent adjacent-segment disease.

Anterior column augmentation with PLIF using intervertebral spacers in addition to PS fixation can produce a superior fusion rate and improve clinical outcomes in spondylolisthesis [12-14]. Recently, Takata et al. [15] proposed a hybrid technique of CBT and pedicle screwing for minimally invasive spine reconstruction surgery. According to the authors, the hybrid CBT-PS technique allows sufficient holding strength of the slipped vertebra compared with other conventional PS procedures. As far as slip reduction and a low percentage of slip loss that is maintained of the initial fixation, our pure CBT screwing for degenerative spondylolisthesis also showed similar good holding power compared with the PS technique.

There was no significant difference between preoperative and postoperative lumbar lordosis in either CBT or conventional PS cases. However, our observations of a slight lordotic change in PS cases and the occurrence of one spacer backout in a CBT case indicates the need 
for further investigation of which technique is better for the maintenance of lordosis. Nevertheless, our results do demonstrate that CBT is well indicated for elderly or osteoporotic patients compared to the conventional PS technique because of its less invasive nature.

This study has some limitations. Most cases were of grade I spondylolisthesis using several different type cages, so we cannot extrapolate to patients with more severe slippage. Second, this study was a small case series. Further investigations with a larger patient populations are needed.

Despite these limitations, CBT screwing showed sufficient holding power in this study. Therefore, pending conformation in future studies, the CBT technique is expected to yield good bone fusion and good long-term clinical outcomes.

\section{Conclusions}

The CBT technique shows similarly good initial fixation compared with the PS procedure for lumbar degenerative spondylolisthesis.

\section{Conflict of Interest}

No potential conflict of interest relevant to this article was reported.

\section{Supplementary Materials}

Suppl. Table 1. \% slip change

Supplemental data can be found at: http://www.asianspinejournal.org/src/sm/asj-10-251-s001.pdf.

Suppl. Table 2. Lumbar lordosis change $\left(^{\circ}\right)$

Supplementary material can be found at: http://www. asianspinejournal.org/src/sm/asj-10-251-s002.pdf.

\section{References}

1. Santoni BG, Hynes RA, McGilvray KC, et al. Cortical bone trajectory for lumbar pedicle screws. Spine J 2009;9:366-73.

2. Matsukawa K, Yato Y, Kato T, Imabayashi H, Asazuma T, Nemoto $\mathrm{K}$. In vivo analysis of insertional torque during pedicle screwing using cortical bone trajectory technique. Spine (Phila Pa 1976) 2014;39: E240-5.
3. Genant HK, Wu CY, van Kuijk C, Nevitt MC. Vertebral fracture assessment using a semiquantitative technique. J Bone Miner Res 1993;8:1137-48.

4. Weinstein JN, Rydevik BL, Rauschning W. Anatomic and technical considerations of pedicle screw fixation. Clin Orthop Relat Res 1992;(284):34-46.

5. Iwatsuki K, Yoshimine T, Ohnishi Y, Ninomiya K, Ohkawa T. Isthmus-guided cortical bone trajectory for pedicle screw insertion. Orthop Surg 2014;6:2448.

6. Daftari TK, Horton WC, Hutton WC. Correlations between screw hole preparation, torque of insertion, and pullout strength for spinal screws. J Spinal Disord 1994;7:139-45.

7. Myers BS, Belmont PJ Jr, Richardson WJ, Yu JR, Harper KD, Nightingale RW. The role of imaging and in situ biomechanical testing in assessing pedicle screw pull-out strength. Spine (Phila Pa 1976) 1996; 21:1962-8.

8. Zdeblick TA, Kunz DN, Cooke ME, McCabe R. Pedicle screw pullout strength. Correlation with insertional torque. Spine (Phila Pa 1976) 1993;18:16736.

9. Law M, Tencer AF, Anderson PA. Caudo-cephalad loading of pedicle screws: mechanisms of loosening and methods of augmentation. Spine (Phila Pa 1976) 1993;18:2438-43.

10. Okuyama K, Abe E, Suzuki T, Tamura Y, Chiba M, Sato K. Can insertional torque predict screw loosening and related failures? An in vivo study of pedicle screw fixation augmenting posterior lumbar interbody fusion. Spine (Phila Pa 1976) 2000;25:858-64.

11. Kawakami M, Tamaki T, Ando M, Yamada H, Hashizume $\mathrm{H}$, Yoshida M. Lumbar sagittal balance influences the clinical outcome after decompression and posterolateral spinal fusion for degenerative lumbar spondylolisthesis. Spine (Phila Pa 1976) 2002;27:5964.

12. Okuyama K, Kido T, Unoki E, Chiba M. PLIF with a titanium cage and excised facet joint bone for degenerative spondylolisthesis--in augmentation with a pedicle screw. J Spinal Disord Tech 2007;20:53-9.

13. Patil SS, Rawall S, Nagad P, Shial B, Pawar U, Nene AM. Outcome of single level instrumented posterior lumbar interbody fusion using corticocancellous laminectomy bone chips. Indian J Orthop 2011;45: 500-3. 
14. Greiner-Perth R, Boehm H, Allam Y, Elsaghir H, Franke J. Reoperation rate after instrumented posterior lumbar interbody fusion: a report on 1680 cases. Spine (Phila Pa 1976) 2004;29:2516-20.

15. Takata Y, Matsuura T, Higashino K, et al. Hybrid technique of cortical bone trajectory and pedicle screwing for minimally invasive spine reconstruction surgery: a technical note. J Med Invest 2014;61:38892. 